\title{
NOTES FROM THE MEDICAL PRESS
}

\author{
IN CHABGE OF \\ ELIZABETH ROBINSON SCOVIL \\ 구구룰
}

ALCOHOL.-The Journal of the American Medical Association gives a synopsis of a paper in the British Medical Journal on this subject as follows: "Hyslop considers that alcohol always has an evil action in healthy individuals, though it may be used wisely in times of need as a stimulant, nutrient, and antipyretic. Stimulating a worn-out machine, however, by improper fuel is not productive of good results. He makes a comparison with financial conditions, and claims that alcohol is only useful as a loan to avert physical bankruptcy. It may help to tide over a bodily crisis until the organism can repay the loan with its accumulated interest. The action of alcohol on the nervous element is noted, as also the changes in alcoholic insanity."

MoIst DRessings-Dr. Charles H. Lemon in the Wisconsin Medical Journal recommends the use of moist dressings in compound fractures. He advises the application of hygroscopic gauze moistened or wrung out of water or an antiseptic solution and applied to the wound. Evaporation and consequent drying is prevented by covering the dressing with oiled silk or gutta-percha tissue. It stimulates the capillary circulation in the region of the wound and induces a vigorous blood-current which mechanically washes it out and brings an abundance of blood-tissue to the part. A for eign contemporary in an article on the after treatment of septic operations says a wet dressing soothes pain far better than a dry one.

Cŕanide of Mercurx as a Sturgical Antiseptic.-Dr. Charles Green Cranston in the New York and Philadelphia Medical Journal advocates the use of cyanide instead of bichloride of mercury as an antiseptic in surgery. His formula is mercury cyanide, one-half gramme; sodium borate (C. P.), one gramme; aniline red, sufficient to color the solution. It does not irritate the tissues and does not coagulate albumen. It does not injure metal when rendered alkaline by the addition of soda. The hands remain soft after its use and the sense of touch perfect. It is not more poisonous than bichloride of mercury, but is a powerful antiseptic. Seventeen milligrammes added to a litre of bouillon prevents the development of micro-organisms. The fact that it does not roughen the hands would recommend it to nurses.

RUBELAA.-Dr. Dillingham in American Medicine believes in scarlet fever, measles, and rubella, but does not believe in the existence of "the fourth disease." The author quotes with approval this saying by Griffith: "The disease (rubella) may be divided into two types, resembling mild measles, and then resembling mild scarlet fever, and then gradations from these types to either extreme: (1) Eruption in which the spots are for the most part nearly or fully the size of split peas, more or less grouped, and having greatest resemblance to measles; (2) rash, confluent in patches, or universally; not elevated, uniform 
redness simulates scarlet fever. Careful investigation often reveals a few papules and general diffuse redness. It is best seen on the wrists and fingers." The author compares in detail the three diseases under discussion, and gives the chief points on which the differential diagnosis must rest. He concludes by saying that to one with large experience in contagious diseases the differential diagnosis of rubella should not usually give much difficulty. But even one with the greatest experience, although he carefully consider every factor, will occasionally fail to make the correct diagnosis in cases where measles is the confusing element."

URotropin IN Scartet Feter.-The Journal of the American Medical Association, quoting from an Austrian exchange, says Widowitz has never had a case of nephritis among one hundred and two scarlet-fever patients since he introduced the plan of preventive administration of urotropin. He gives from 0.05 gramme to 0.5 gramme of urotropin three times a day according to age during the first three days of the disease, and again at the beginning of the third week for another three days.

DeeP Breathing.-In a paper in the New York and Philadelphia Medical Journal Dr. Richard Cole Newton says that a physical culturist after measuring the lung capacity of twenty thousand persons found that not one per cent. of them had strong lungs and breathed fully. He advises the practice of full inflation of the lungs by slow, deep, regular breathing continued until it becomes habitual, and considers it of great benefit as a prophylactic in phthisis. He sums up his conclusions as follows: (1) Deep breathing is essential to good health, and is in many cases a valuable therapeutic measure. (2) Its importance is not at all generally appreciated. (3) It should be a part of every child's education. (4) It is especially indicated for backward and sickly children. (5) The profession owes it to itself to study more deeply this vital question and to be able to instruct the laity fully upon all its bearings.

Disinfection of the Stools in Contagious Diseases.-Revue frangaise de médecine et de chirurgie advises the following antiseptic mixture: Zine sulphate, one hundred grammes (twenty-five drachms); sulphuric acid, ten grammes (two and one-half drachms); benzaldehyde, two centigrammes (three-tenths grain); indigo, fifteen centigrammes (two and one-quarter grains). Drop seventy-five minims into vessel before using. The indigo is added merely to give a distinctive color.

The Handkerchief as a Disseminator of Disease.-The Medical Record comments on the New York Times's account of a meeting of the National Woman's Temperance Union held in Cincinnati, where handkerchief shaking as a method of salute was warmly discussed from a hygienic standpoint. Miss Marie Brehm, of Illinois, declared that the fluttering of innumerable handkerchiefs in welcome to a speaker was quite sufficient to account for the wide distribution of colds among those attending the convention. Miss Brehm moved that handkerchiefs be kept in the pocket, where they belong, and the saluting be done with little silk flags provided for the purpose. It is, in fact, a grave sanitary question whether the handkerchief does not do more harm than good as it is ordinarily used. When we assume that the healthy nose does not need to be wiped fwe face 
a reasonably broad proposition as to the danger of the handkerchief as a disease propagator. Most nasal catarrhs are of an infectious character, notably those of grip origin. Contrary to a general law of asepsis, the handkerchief saturated with disease germs, instead of being promptly washed, is stowed for hours in the pocket, with a result that can be easily imagined. Is it any wonder, then, that catarrhs are constantly fostered by a system of autoinfection? Even when the discharges become dried upon this dangerous article of the toilet, its mere handling or use by others must necessarily be a means of conveying infection. Worse than the nasal discharges are the expectorations, which so often thus find their way into the pocket.

Cure of Sciatica.-The New York and Philadelphia Medical Journal, quoting from a Spanish contemporary, says: "Castelvi has used subeutaneous injections of pure oxygen in five cases with excellent results. The author gives a detailed description and illustration of the apparatus used. Injections of from two hundred to one thousand cubic centimetres of the gas were made in the gluteal region of the affected side, the injections being repeated daily till all pain subsided. This result was accomplished in from one to five treatments. The immediate effect of each injection was the subsidence of pain either in the whole course of the nerve or over an area extending considerably beyond the limits of the injected part. Alleviation of pain was experienced in many instances during the injection. No discomfort was experienced by the patients so treated and the gas was quite rapidly absorbed. In some cases the pain permanently disappeared after one injection."

Adenoids and Nocturnat Incontinence.-The New York and Philadelphia Medical Journal has a synopsis of an article in a foreign exchange as follows: "Melzi reminds us that adenoids in the pharynx are in some way connected with nocturnal incontinence in children. Various theories have been advanced to explain this connection, but the fact remains that the removal of the adenoid growths causes a cessation of the incontinence. The author reports two striking cases, and advises the practical physician to think of adenoids whenever he meets a case of nocturnal incontinence."

Caffexine IN Alcoholism.-Hall, in the Medical News, considers that caffeine is almost a specific in alcoholic toxæmia. He asserts that this drug, in doses of one to two grains every one, two, or three hours, will usually, in from twenty-four to forty-eight hours, quench the thirst or craving for alcohol to such an extent that the most confirmed habitues will voluntarily abandon its use. Four cases are reported which seem to uphold the author's contention.

FormatDehyde Test for ALbUMen IN URINE.-The Journal of the American Medical Association, quoting from an Italian contemporary, says Introna has found that formaldehyde causes the coagulation of albumen in solution, and recommends a new test for albuminuria based on this fact. He adds one cubic centimetre of formaldehyde to fifty-six cubic centimetres of urine. If it is free from albumen, the fluid remains clear, but if albumen is present, it becomes turbid and a flocculent precipitate is gradually deposited consisting entirely of the albumen. The test can be hastened by heating to boiling-point, in which case the precipitate is deposited without delay. 